\title{
Outbreaks of Mumps Genotype G Viruses in the Netherlands Between October 2019 and March 2020: Clusters Associated With Multiple Introductions
}

\section{Anita Shah ( $\nabla$ anita.shah@rivm.nl)}

Center for Infectious Disease Control, National Institute for Public Health and Environment (RIVM), Bilthoven, The Netherlands

\section{Rogier Bodewes}

Center for Infectious Disease Control, National Institute for Public Health and Environment (RIVM), Bilthoven

\section{Linda Reijnen}

Center for Infectious Disease Control, National Institute for Public Health and Environment (RIVM), Bilthoven

\section{Timo Boelsums}

Department of Infectious Disease Control, Public Health Service Rotterdam-Rijnmond (GGD), Rotterdam

\section{Claudia Weller}

Department of Infectious Disease Control, Public Health Service Rotterdam-Rijnmond (GGD), Rotterdam

\section{Ewout Fanoy}

Department of Infectious Disease Control, Public Health Service Rotterdam-Rijnmond (GGD), Rotterdam Irene Veldhuijzen

Center for Infectious Disease Control, National Institute for Public Health and Environment (RIVM), Bilthoven

\section{Research Article}

Keywords: MMR, mumps, surveillance, genotype G viruses, the Netherlands

Posted Date: January 20th, 2021

DOI: https://doi.org/10.21203/rs.3.rs-126893/v1

License: (c) (1) This work is licensed under a Creative Commons Attribution 4.0 International License. Read Full License 


\section{Abstract}

\section{Background}

From October 2019-March 2020, several clusters of mumps cases were identified in the Netherlands. Our objective was to describe cluster-associated mumps virus transmission using epidemiological and molecular information in order to help future mumps prevention efforts.

\section{Methods}

An epidemiological cluster includes $\geq 2$ mumps cases with at least an epidemiological-link to a laboratory-confirmed mumps case. A molecular group includes $\geq 2$ mumps cases with identical mumps virus sequences. Cases with symptom onset date between 1 October 2019-31 March 2020 reported through the National Notifiable Diseases Surveillance System were included. We described epidemiological and clinical characteristics of mumps cases. Sequence data was obtained from selected regions of mumps virus genomes (2270 nucleotides). Correlations between epidemiological and molecular information were investigated.

\section{Results}

In total, 102 mumps cases were notified (90\% laboratory-confirmed, 10\% epidemiologically-linked). Fourteen epidemiological clusters were identified containing 46 (range 2-12) cases. Complete sequence data was obtained from 50 mumps genotype $\mathrm{G}$ viruses. Twelve molecular groups were identified containing 43 (range 2-13) cases, dispersed geographically and timewise. Combined information redefined 7 epidemiological clusters into 2 distinct molecular groups. The first lasting for 14 weeks, the other for 6 . Additionally, one molecular group was detected, linked by geography and time but without an epidemiological-link.

\section{Conclusions}

Combined epidemiological and molecular information indicated ongoing mumps virus transmission from multiple introductions for extended time periods. Sequence analysis provided valuable insights into epidemiological clustering. If combined information is available real-time, this would improve outbreak detection, generate further insight into mumps transmission, and guide necessary control measures.

\section{Background}

Mumps is an acute infectious disease caused by a paramyxovirus and is usually spread human-tohuman by direct contact or via airborne droplets. The incubation period is $16-18$ days and the infectious period commences 5 days prior and up to 9 days following the onset of symptoms. Mumps can usually be characterised by parotitis (inflammation of the salivary glands) and the disease is usually mild, however, complications can occur which may include meningitis, orchitis or encephalitis [1]. 
The combination vaccine against measles, mumps, and rubella (MMR) was first introduced in the Netherlands in 1987 for all children aged 14 months and 9 years as part of the Dutch National Immunisation Program [2]. Following the introduction of the MMR vaccination program, the incidence of mumps decreased in the Netherlands, however, several mumps outbreaks have been detected since then. In 2004, an outbreak occurred at an international hotel school [3], and in 2007-2008, an outbreak occurred predominantly in a religious community that had a low vaccination coverage [4]. The largest outbreaks since then have occurred at the end of 2009 to 2012, mainly affecting student populations with a high vaccination coverage $[5,6]$. Explanations for these outbreaks could be the possibility of waning immunity in individuals who are appropriately immunised [7]. Other factors may include insufficient effectiveness of the mumps component of the MMR vaccine or increased potential transmission via crowded spaces and social gatherings $[7,8]$.

Between 2013 and 2019, the maximum number of reported mumps cases in one year was 131 (2019). However, there is likely undetected circulation of mumps virus in the Netherlands. This may be due to several reasons such as patients may be asymptomatic or present with milder symptoms of mumps and thus, do not seek health care [9]. Even if symptomatic patients do seek health care at the general practitioner, laboratory testing may not be conducted for all cases and therefore, it is highly probable that mumps cases may be missed.

From October 2019 to March 2020, several clusters of mumps cases were reported to the National Institute for Public Health and Environment (RIVM) in the Netherlands. The first alert regarding an ongoing mumps cluster was received on 25 October and the identified potential source case was reported to have attended a party held on 4 October while symptomatic. In the following weeks, 11 additional mumps cases were reported as part of this cluster with either epidemiological or molecular sequencing links. Subsequently, additional mumps cases were reported from other close-contact settings. As the number of cases was limited, this offered a unique opportunity to analyse these cases. Our primary objective was to identify and describe clusters of associated mumps virus transmission from multiple exposures by using epidemiological information alone, molecular surveillance alone, and using both together. Our secondary objective was to assess whether mumps cases were occurring due to ongoing transmission or from repeated introductions of genetically distinct mumps virus.

\section{Methods}

\section{Case definitions}

In the Netherlands, mumps is a notifiable disease under the Dutch Public Health Act [10]. Mumps cases are reported to the national registration system for notifiable diseases (OSIRIS) by the Municipal Health Service who receives the information from the clinicians and medical microbiology laboratories [11]. The notification criteria for a mumps case includes at least one related symptom (acute onset of painful swelling of the parotid or salivary glands, orchitis or meningitis) and laboratory confirmation of infection or an epidemiological link to a laboratory-confirmed case [6]. 
An epidemiological cluster includes 2 or more cases who met the notification criteria and had an epidemiological link to a confirmed mumps case.

A molecular group includes 2 or more mumps cases and in which mumps viruses were detected with identical sequence data that was different from other molecular variants detected in the same time period.

\section{Epidemiological analysis}

We reviewed data on mumps cases reported to OSIRIS with a date of symptom onset between 1 October 2019 to 31 March 2020 in the Netherlands. Using available information, we performed descriptive analyses of all cases in terms of demographic characteristics, geographical location, import status, vaccination status, mumps complications and hospitalisation status. R software version 4.0.2 was used for statistical analyses and visualisation of epidemiological data.

\section{Molecular analysis}

Clinical specimens (oral fluid, throat swab and/or urine) from suspected or laboratory confirmed mumps cases with date of onset between 1 October 2019 and 31 March 2020 were submitted to the RIVM for molecular diagnostics and/or for molecular surveillance [12]. Clinical samples were tested for the presence of mumps virus RNA using real time quantitative PCR as described previously [13]. Samples in which mumps virus RNA was detected, were subject to sequencing of the $\mathrm{SH}$ gene and the non-coding regions between the $N$ and $P, P$ and $M$ and $M$ and $F$ genes as described previously [14, 15]. Sanger sequencing was performed at BaseClear (Leiden, the Netherlands). Obtained sequences were manually checked in Bionumerics version 7.6.3 and subsequent phylogenetic analyses were performed on concatenated sequences with UPGMA (unweighted pair group method with arithmetic mean) using Bionumerics version 7.6.3 [16]. In addition, a phylogenetic tree was constructed using IQ-tree software via the webserver (W-IQ-TREE) [16-18] with the maximum likelihood method and the transition model + $F$ $(\mathrm{TIM}+\mathrm{F})$ model according to bayesian information criterion (BIC) based on analysis with ModelFinder [19]. Branch support was calculated using the ultrafast bootstrap approach (UFboot) with 1000 bootstrap alignments [20] and the phylogenetic tree was visualised using FigTree v1.4.4 [21]. Mumps virus MuV/lowa/6/06 (JX287385) was used as reference strain in the phylogenetic analysis. Molecular surveillance of mumps viruses, including this study, is part of the Public Health Act in the Netherlands.

\section{Results}

\section{Epidemiological analysis}

Between 1 October 2019 and 31 March 2020, 102 mumps cases were notified with a date of onset within this period. Of these, 92 (90\%) were laboratory-confirmed, and 10 (10\%) were epidemiologically-linked. The median age of all cases was 26 years (range: $3-71$ years). Of all cases, 57 (56\%) were male and 31 (31\%) were students. For 97 out of 102 (95\%) cases, the vaccination status was known. Of those, 58 
(60\%) cases had received two or more MMR doses, 14 (15\%) one dose, $4(4 \%)$ were vaccinated with number of doses unknown, and 21 (21\%) were unvaccinated. Of the 21 unvaccinated cases, the median age was 35 years old (range 3-71). Two patients, aged 21 and 44 years, and vaccinated with two doses, were hospitalised; both reported orchitis. Among the cases not hospitalised, 5 cases reported orchitis, and 1 case reported an eye infection. Nineteen cases (19\%) acquired the infection abroad and country of infection was unknown for 7 cases (7\%).

Forty-six of the 102 mumps cases were identified to be part of 14 epidemiological clusters (Table 1, Figure 1). All 14 epidemiological clusters were identified using epidemiological information alone. The median age among epidemiological cluster-associated cases was 25.5 years (range 3-71 years). Of all cases, 30 (65\%) were male. All of the identified epidemiological clusters contained some close-contact involvement and settings included contact at a party, secondary schools, football match, hotels, and sharing the same household (Table 1).

Two of 14 epidemiological clusters had 2 co-primary cases in each with the same earliest date of symptom onset as the initial source case of their cluster was not identified. The median age of the 16 source cases of each cluster was 22.5 years (range 5-42 years), 15 cases were vaccinated, and 6 acquired the infection abroad. The place of infection of the source case or co-primary case of the cluster infected abroad was in either Western Europe, Central Europe, or North America (Table 1).

The first identified and largest epidemiological cluster (2019-7) occurred in the provinces South Holland and North Holland, including 12 cases (Table 1, Figure 1A). The index case had attended a party and was also working at a secondary school. Thereafter, secondary and tertiary cases occurred among attendees of the party and their partners as well as among 4 staff and 1 student at the secondary school. The second epidemiological cluster (2019-8) occurred among attendees of a football match in South Holland and Gelderland. Four additional epidemiological clusters occurred in South Holland (2019-9, 2019-10, 2019-11, and 2019-12). Two of these clusters occurred in school settings, and the other 2 occurred among family members.

For the remaining 8 epidemiological clusters, all contained transmission in close-contact settings among family, friends, or partners (Table 1). Geographically, 4 of these epidemiological clusters (2020-1, 2020-5, 2020-6, and 2020-7) contained cases reporting onset of symptoms in North Holland. One of the clusters (2020-5) contained cases reporting onset of symptoms in North Holland and Utrecht provinces as they attended a winter sports trip together in another location and returned to their respective provinces of residence. One epidemiological cluster (2020-3) occurred among partners in Zeeland who had travelled abroad. Two epidemiological clusters occurred in Groningen (2020-2 and 2020-4); the first occurring among attendees of a swimming club and their family and the second occurring among 2 persons travelling on holiday together. The final epidemiological cluster (2020-8) occurred in Gelderland province among family members.

\section{Molecular surveillance}


Using sequence data from the $\mathrm{SH}$ gene, a genotype could be obtained from 59 out of 60 mumps cases from which one or more clinical materials were submitted to the RIVM. In 58 cases, a mumps genotype $G$ virus was detected, while in 1 case a mumps genotype $C$ virus was detected. Complete NCRs sequence data could be obtained from 50 mumps genotype $G$ viruses (Genbank accession numbers MW006669$M W 006820$ ). Sequence analysis of these mumps genotype $G$ viruses revealed that at 30 nucleotide positions within the $\mathrm{SH}+\mathrm{NCR}$ sequences nucleotide variation was present, which resulted in 19 different molecular variants ( $A$ to $S$ ) with one or more viruses that had at least one nucleotide difference compared to other mumps viruses (Figure 1C, Supplementary Figure S1). From reviewing the 19 molecular variants, 12 of these contained 2 or more cases. Therefore, 12 molecular groups were identified according to the definition including 43 mumps viruses in total.

\section{Comparison of results of epidemiological analysis and molecular surveillance}

Molecular group A contained the highest number of mumps cases (13); ten from 4 different epidemiological clusters and 3 individual cases (Figure 1C). Combined information redefined 4 epidemiological clusters into 1 distinct molecular group. All 13 cases were shown to be dispersed over time with dates of symptom onset occurring over 14 weeks in total, as well as geographically with the majority from South Holland (12/13), and Gelderland provinces (1/13). Three of the 13 cases were not identified as part of an epidemiological cluster.

The second biggest molecular group E contained 6 cases; four from 3 different epidemiological clusters and 2 individual cases (Figure 1C). Combined information redefined 3 epidemiological clusters into 1 distinct molecular group. Cases had dates of symptom onset over a duration of 6 weeks in total, and cases occurred in 4 different provinces, Gelderland, North and South Holland, and Utrecht.

Four molecular groups ( $D, M, R$, and $S$ ) each contained 3 mumps viruses with sequence data. Molecular group $\mathrm{R}$ contained cases linked by geography and time but without an epidemiological-link. Molecular group $S$ contained 3 cases from the same epidemiological cluster and cases had dates of symptom onset over a duration of 2 weeks. Molecular group M contained 2 cases from the same epidemiological cluster and 1 individual case. Molecular cluster D contained 1 case from an epidemiological cluster and 2 individual cases.

The remaining 6 molecular groups each contained mumps viruses detected in 2 cases. Only 1 of these molecular groups contained 2 cases from the same epidemiological cluster $(\mathrm{J})$, while the rest only included individual cases.

Table 1. Characteristics of epidemiological clusters, the Netherlands, 1 October 2019-31 March 2020 ( $n=$ 46 cases) 


\begin{tabular}{|c|c|c|c|c|}
\hline $\begin{array}{l}\text { Epidemiological } \\
\text { cluster }\end{array}$ & Cases & $\begin{array}{l}\text { Province of } \\
\text { symptom onset }\end{array}$ & $\begin{array}{l}\text { Description } \\
\text { of setting }\end{array}$ & $\begin{array}{l}\text { Place of infection of source or co- } \\
\text { primary case (if abroad) }\end{array}$ \\
\hline 2019-7 & 12 & $\begin{array}{l}\text { South Holland, } \\
\text { North Holland }\end{array}$ & $\begin{array}{l}\text { Party, } \\
\text { secondary } \\
\text { school }\end{array}$ & \\
\hline 2019-8 & 4 & $\begin{array}{l}\text { South Holland, } \\
\text { Gelderland }\end{array}$ & $\begin{array}{l}\text { Football } \\
\text { match }\end{array}$ & \\
\hline 2019-9 & 2 & South Holland & $\begin{array}{l}\text { Secondary } \\
\text { school }\end{array}$ & \\
\hline 2019-10 & 3 & South Holland & Hotel, family & \\
\hline 2019-11 & 2 & South Holland & School & \\
\hline $2019-12$ & 2 & South Holland & Family & \\
\hline 2020-1 & 2 & North Holland & Friends & Western Europe \\
\hline $2020-2$ & 4 & Groningen & $\begin{array}{l}\text { Swimming } \\
\text { club, family }\end{array}$ & \\
\hline $2020-3$ & 2 & Zeeland & Partners & Western Europe \\
\hline $2020-4$ & 2 & Groningen & $\begin{array}{l}\text { Holiday } \\
\text { abroad }\end{array}$ & Central Europe \\
\hline $2020-5$ & 2 & $\begin{array}{l}\text { North Holland, } \\
\text { Utrecht }\end{array}$ & $\begin{array}{l}\text { Winter sport } \\
\text { trip abroad }\end{array}$ & Western Europe \\
\hline $2020-6$ & 3 & North Holland & Family & \\
\hline $2020-7$ & 3 & North Holland & $\begin{array}{l}\text { Partners and } \\
\text { friend }\end{array}$ & North America \\
\hline $2020-8$ & 3 & Gelderland & Family & \\
\hline
\end{tabular}

\section{Discussion}

Our primary objective was to describe mumps cases using epidemiological and molecular sequencing information. In this retrospective study, 21 of 71 (30\%) epidemiologically and/or molecularly-associated cases were identified solely through epidemiological information, 25 (35\%) identified solely from molecular surveillance, and 25 (35\%) identified using both. Overall, molecular surveillance redefined 7 different epidemiological clusters into 2 distinct molecular clusters and our findings indicated ongoing mumps virus transmission for extended time periods.

The largest molecular group (molecular variant A) contained 13 cases from 4 different epidemiological clusters and the second largest (molecular variant E) contained cases from 3 different epidemiological 
clusters. The 2 molecular groups each lasted for an extended time period of 14 and 6 weeks, respectively. As molecular clustering together with epidemiological clustering reflects time passed between clusters, this indicated ongoing transmission of mumps virus of the same molecular variant [22]. In this instance, molecular surveillance demonstrated an extended time period of transmission within the cluster than would have otherwise been determined solely from epidemiological information. Molecular surveillance has been shown to discriminate distinct lineages within individual transmission events and in outbreaks for various paramyxoviruses $[23,24]$.

Of interest is the molecular group containing 3 cases with molecular variant R. Cases were closely related by time as the date of symptom onset between cases matched the known incubation period for mumps (16-18 days) [1]. They had a similar geographical location as all were reported from Limburg province, however, no common source or epidemiological link was identified. In this particular instance, molecular sequencing provided valuable information in the absence of a clear epidemiological link and we identified a molecular group which would not have been otherwise determined from epidemiological information [24].

Three molecular groups (molecular variants $\mathrm{G}, \mathrm{N}$, and 0 ) each contained 2 individual cases where 1 of the cases was infected abroad. This is particularly interesting as for each group, the 2 cases do not have a similar geographical location and the time between their symptom onset is not reflective of the known incubation period of mumps [1]. Therefore, these results suggest that mumps viruses with identical $\mathrm{SH}+\mathrm{NCR}$ sequences are circulating at the same time in different countries and/or regions. Using sequences from known data repositories to compare outbreak strains helps in distinguishing potential international transmission events, which can then be correlated with available epidemiological information [24].

In the largest epidemiological cluster in South and North Holland province $(2019-7, n=12)$ which lasted 6 weeks, molecular variant A was identified in 4 out of 5 cases where molecular sequencing was available. No additional cases were reported after week 45 with epidemiological links to the party or school according to epidemiological information. This may have been due to existing events which naturally hindered additional cases from occurring such as a mid-term holiday and winter holidays at the school. This would have reduced the exposure of susceptible persons from mumps cases. Subsequently, this cluster did not warrant large scale control measures and the prevention strategy in place included targeted communication to groups at risk. However, identical $\mathrm{SH}+\mathrm{NCR}$ sequences with one nucleotide variation were identified for mumps viruses detected in all 6 epidemiological clusters reported from South Holland (2019-7, 2019, 8, 2019, 9, 2019-10, 2019-11, and 2019-12). The similar molecular sequencing may be explained by geographical proximity of the cases. As a number of individual cases were not identified as part of an epidemiological cluster but contained the same molecular variant undetected transmission of mumps is most likely present. Similar situations whereby a cluster with missing epidemiological information has been redefined by molecular sequencing has been described previously [22]. 
Several limitations exist that should be considered when analysing clusters of mumps cases using epidemiological and molecular information at the national level. Molecular sequences available in this study may not be representative for all mumps virus strains circulating during the study period. This can

be due to several reasons as there is likely underreporting of mumps cases as individuals with symptoms are only notified in the surveillance system if they are laboratory-confirmed or if they have an epidemiological link in the Netherlands. Patients who are asymptomatic and/or who are vaccinated with two or more doses may present with milder symptoms and thus, do not seek health care and there is limited active case finding [25]. Even when patients do visit their general practitioner, laboratory testing may not be conducted for cases and therefore, mumps cases are not reported in the surveillance database. Epidemiological links may be missing from the national surveillance system and hence, it is difficult to determine which cases are part of a specific cluster solely from reviewing the epidemiological information. Thirty-one cases (30\%) of the 102 cases had no available epidemiological or molecular information and were dispersed timewise and geographically. If molecular variant information had been available for these cases, this would have provided useful insights in circulating lineages, particularly when there were several epidemiological clusters and molecular variants present, such as between weeks 3 and 13.

\section{Conclusions}

In conclusion, combined epidemiological and molecular information demonstrated ongoing mumps virus transmission for extended time periods. Sequence analysis contributes to surveillance of mumps cases to show the change of the variants over time as well as to provide valuable insights in epidemiological clustering. These findings illustrate the importance of combining epidemiological and molecular sequencing information in mumps cluster identification. Cluster information including geographical and temporal distribution of mumps genotypes that is available real-time, improves outbreak detection and guides implementation of any necessary control measures. In addition, it generates further clarity of mumps transmission patterns. This combined information can improve targeted public health recommendations and guidance including testing advice and social distancing while being symptomatic.

\section{Abbreviations}

BIC: Bayesian information criterion

MMR: Measles, mumps, rubella vaccine

OSIRIS: National registration system for notifiable diseases

PCR: polymerase chain reaction

RIVM: National Institute for Public Health and Environment

UFboot: Ultrafast bootstrap approach 
UPGMA: Unweighted pair group method with arithmetic mean

\section{Declarations}

\section{Ethics approval and consent to participate}

Molecular surveillance of mumps viruses, including this study, is part of the Public Health Act in the Netherlands. Therefore, no informed consent was required for this study using anonymised routine surveillance data.

\section{Consent for publication}

Not applicable.

\section{Availability of data and materials}

The datasets generated during and/or analysed during the current study are not publicly available but are available from the corresponding author on reasonable request. The mumps virus sequences, including metadata, collected for this study are available in GenBank, accession numbers: MW006669-MW006820.

\section{Competing interests}

The authors declare that they have no competing interests.

\section{Funding}

No external funding was received.

\section{Authors' contributions}

AS led on the data analysis and drafted the manuscript. RB and LR conducted molecular analyses. RB, $L R, T B, C W, E F$, and IV were involved in the interpretation of the data. All authors critically revised the manuscript and approved its final version.

\section{Acknowledgements}

We thank staff at Municipal Health Services, laboratories and clinicians for reporting and investigating mumps cases and for sending clinical materials of mumps patients to the RIVM for genotyping. We also acknowledge colleagues from RIVM in sequence analyses. Moreover, we thank the contributions of Sooria Balasegaram (Public Health England) and Mirjam Knol (RIVM) for their useful and constructive suggestions on this paper.

\section{References}

1. Hviid A, Rubin S, Mühlemann K. Mumps. Lancet. 2008;371(9616):932-44. 
2. van den Hof S, Beaumont MT, Berbers GA, de Melker HE. Antibodies against mumps in The Netherlands as assessed by indirect ELISA and virus neutralization assay. Epidemiol Infect. 2003;131(1):703-9.

3. Brockhoff HJ, Mollema L, Sonder GJ, Postema CA, van Binnendijk RS, Kohl RH, et al. Mumps outbreak in a highly vaccinated student population, The Netherlands, 2004. Vaccine. 2010;28(17):2932-6.

4. Snijders BE, van Lier A, van de Kassteele J, Fanoy EB, Ruijs WL, Hulshof F, et al. Mumps vaccine effectiveness in primary schools and households, the Netherlands, 2008. Vaccine. 2012;30(19):29993002.

5. Greenland K, Whelan J, Fanoy E, Borgert M, Hulshof K, Yap KB, et al. Mumps outbreak among vaccinated university students associated with a large party, the Netherlands, 2010. Vaccine. 2012;30(31):4676-80.

6. Whelan J, van Binnendijk R, Greenland K, Fanoy E, Khargi M, Yap K, et al. Ongoing mumps outbreak in a student population with high vaccination coverage, Netherlands, 2010. Euro Surveill. 2010;15(17).

7. Kennedy RB, Ovsyannikova IG, Thomas A, Larrabee BR, Rubin S, Poland GA. Differential durability of immune responses to measles and mumps following MMR vaccination. Vaccine. 2019;37(13):177584.

8. Hahné S, Schurink T, Wallinga J, Kerkhof J, van der Sande M, van Binnendijk R, et al. Mumps transmission in social networks: a cohort study. BMC Infect Dis. 2017;17(1):56.

9. Dittrich S, Hahné S, van Lier A, Kohl R, Boot H, Koopmans M, et al. Assessment of serological evidence for mumps virus infection in vaccinated children. Vaccine. 2011;29(49):9271-5.

10. RIVM. Vaccination rate and annual report National Vaccination Program Netherlands 2018.

11. RIVM. Mumps 2020 [Available from: https://lci.rivm.nl/richtlijnen/bof.

12. Uchida K, Shinohara M, Shimada S, Segawa Y, Doi R, Gotoh A, et al. Rapid and sensitive detection of mumps virus RNA directly from clinical samples by real-time PCR. J Med Virol. 2005;75(3):470-4.

13. Bodewes R, Reijnen L, Kerkhof J, Cremer J, Schmitz D, van Binnendijk R, et al. Molecular epidemiology of mumps viruses in the Netherlands, 2017-2019. PLoS One. 2020;15(9):e0233143.

14. Gavilán AM, Fernández-García A, Rueda A, Castellanos A, Masa-Calles J, López-Perea N, et al. Genomic non-coding regions reveal hidden patterns of mumps virus circulation in Spain, 2005 to 2015. Euro Surveill. 2018;23(15).

15. Bodewes R, van Rooijen K, Cremer J, Veldhuijzen IK, van Binnendijk R. Optimizing molecular surveillance of mumps genotype G viruses. Infect Genet Evol. 2019;69:230-4.

16. Kumar S, Stecher G, Tamura K. MEGA7: Molecular Evolutionary Genetics Analysis Version 7.0 for Bigger Datasets. Mol Biol Evol. 2016;33(7):1870-4.

17. Nguyen LT, Schmidt HA, von Haeseler A, Minh BQ. IQ-TREE: a fast and effective stochastic algorithm for estimating maximum-likelihood phylogenies. Mol Biol Evol. 2015;32(1):268-74. 
18. Trifinopoulos J, Nguyen LT, von Haeseler A, Minh BQ. W-IQ-TREE: a fast online phylogenetic tool for maximum likelihood analysis. Nucleic Acids Res. 2016;44(W1):W232-5.

19. Kalyaanamoorthy S, Minh BQ, Wong TKF, von Haeseler A, Jermiin LS. ModelFinder: fast model selection for accurate phylogenetic estimates. Nat Methods. 2017;14(6):587-9.

20. Minh BQ, Nguyen MA, von Haeseler A. Ultrafast approximation for phylogenetic bootstrap. Mol Biol Evol. 2013;30(5):1188-95.

21. Rambaut A. FigTree v1.4.4. 2018.

22. Wohl S, Metsky HC, Schaffner SF, Piantadosi A, Burns M, Lewnard JA, et al. Combining genomics and epidemiology to track mumps virus transmission in the United States. PLoS Biol. 2020;18(2):e3000611.

23. Gardy JL, Naus M, Amlani A, Chung W, Kim H, Tan M, et al. Whole-Genome Sequencing of Measles Virus Genotypes H1 and D8 During Outbreaks of Infection Following the 2010 Olympic Winter Games Reveals Viral Transmission Routes. The Journal of Infectious Diseases. 2015;212(10):1574-8.

24. Stapleton PJ, Eshaghi A, Seo CY, Wilson S, Harris T, Deeks SL, et al. Evaluating the use of whole genome sequencing for the investigation of a large mumps outbreak in Ontario, Canada. Sci Rep. 2019;9(1):12615.

25. Gouma S, Cremer J, Parkkali S, Veldhuijzen I, van Binnendijk RS, Koopmans MPG. Mumps virus F gene and $\mathrm{HN}$ gene sequencing as a molecular tool to study mumps virus transmission. Infect Genet Evol. 2016;45:145-50.

\section{Figures}


A.

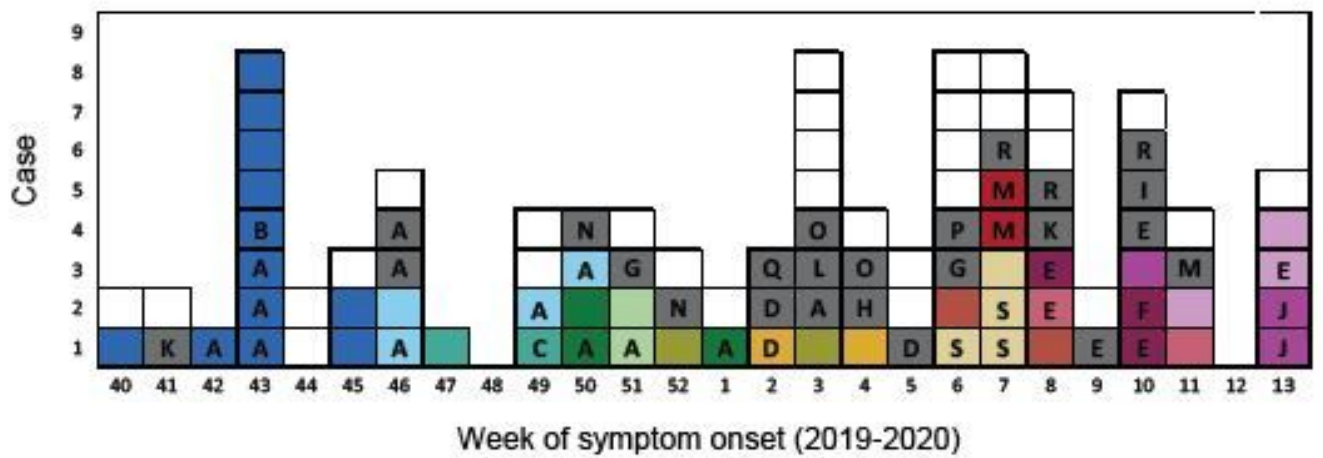

B.

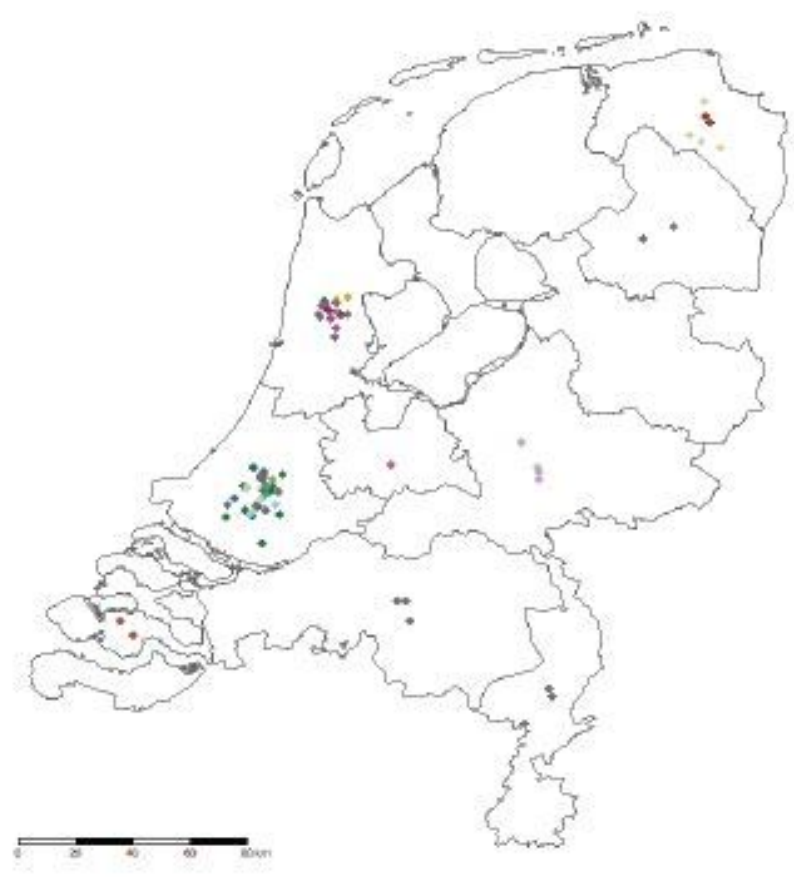

C.

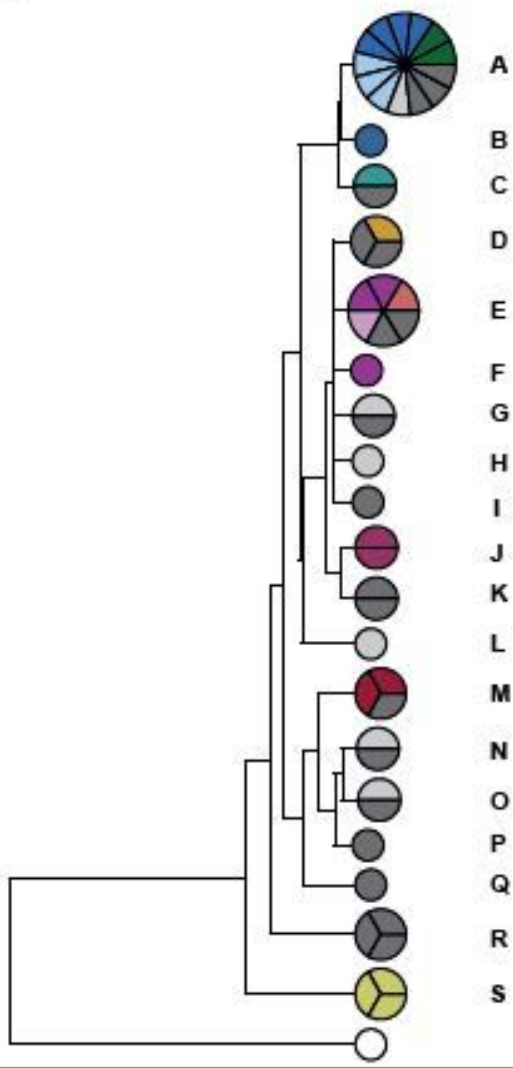

Reference strain Import case with molecular variant Individual case with molecular variant

\section{Figure 1}

A. Mumps cases by week of symptom onset between 1 October 2019-31 March 2020, the Netherlands (n $=100$ cases) ${ }^{\star}$. Figure 1B. Epidemiological cluster (2019-7 to 2020-8) and molecular group associated cases by province between 1 October 2019-31 March 2020, the Netherlands ( $\mathrm{n}=71 \mathrm{cases}){ }^{\star \star}$. Figure 1C. Phylogenetic tree of molecular variants (A-S) of mumps genotype $\mathrm{G}$ viruses detected between 1 October 2019-31 March 2020, the Netherlands ( $=50$ cases). *Two individual cases are not shown in Figure $1 \mathrm{~A}$ 
as date of onset is not available; one of these cases contains molecular variant $\mathrm{C}$. **For confidentiality reasons, the points in Figure 1B have been jittered. Note: The designations employed and the presentation of the material on this map do not imply the expression of any opinion whatsoever on the part of Research Square concerning the legal status of any country, territory, city or area or of its authorities, or concerning the delimitation of its frontiers or boundaries. This map has been provided by the authors.

\section{Supplementary Files}

This is a list of supplementary files associated with this preprint. Click to download.

- Supplementaryfigure.pdf 